\title{
Electrochemically Gated Graphene Field-Effect Transistor for Extracellular Cell Signal Recording
}

\author{
Sanaz Asgarifar ${ }^{1,2}$, Henrique L. Gomes ${ }^{1,2}$, Ana Mestre ${ }^{1,2}$, Pedro Inácio ${ }^{1,2}, \mathrm{~J}$. \\ Bragança $^{1}$, Jérôme Borme ${ }^{3}$, George Machado Jr. ${ }^{3,4}$, Fátima Cerqueira ${ }^{3,4}$ and Pedro \\ Alpuim ${ }^{3,4}$, \\ ${ }^{1}$ Universidade do Algarve, FCT, Faro, Portugal. \\ ${ }^{2}$ IT-Instituto de Telecomunicações, Av. Rovisco, Pais, 1, Lisboa, Portugal. \\ ${ }^{3} \mathrm{INL}$ - International Iberian Nanotechnology Laboratory, Av. Mestre José Veiga, Braga, \\ Portugal \\ ${ }^{4}$ CFUM - Centre of Physics of the University of Minho, Campus de Gualtar, 4710-057 \\ Braga, Portugal \\ sanaz.asgarifar@gmail.com
}

\begin{abstract}
This work presents an experimental characterization of electrochemically gated graphene field-effect transistors (EGFETs) to measure extracellular cell signals. The performance of the EGFETs was evaluated using cardiomyocytes cells. Extracellular signals with a peak value of 0.4 pico-amperes (pA) embedded in a noise level of $0.1 \mathrm{pA}$ were recorded. Signals in current mode were compared with signals recorded as a voltage. Signals below $28 \mu \mathrm{V}$ of magnitude can be detected in a noise floor of $7 \mu \mathrm{V}$ with a signal-to-noise ratio of 4.
\end{abstract}

Keywords: Graphene, Field effect transistor, extra-cellular cell signal recording.

\section{Introduction}

Bioelectronic devices fabricated with emergent materials are currently being developed to establish electrical interfaces with living cells and tissues. The aim is to develop transducers that can record extracellular signals in complex biological environments [1-9]. In contrast with conventional semiconductors such as silicon some of these materials can be deposited into flexible, conformable and biocompatible substrates. Graphene deposited in thin layers is particular interesting for implantable biomedical devices because it offers a high electronic performance combined with biocompatibility and processing on a variety of flexible substrates [10-14].

The recent efforts in improving the fabrication of thin-layers of graphene have made possible to fabricate bioelectronic sensors know as electrochemically-gated field effect transistor (EGFETs). Although, these are field effect devices, unlike conventional transistors do not have a built-in dielectric layer. The gate dielectric is established when the device is immersed into the electrolyte solution. This occurs because when conductive or semiconductive materials are immersed into electrolytes a Helmholtz capacitive double-layer is established at the material/electrolyte interface. This type of device has been used to record extracellular electrophysiological signals from cells [15- 
16].

In this paper, we show that a field effect transistor based in electrochemically graphene gate can be used for extracellular signal recording. The aim is to demonstrate the detection limit of these sensing devices. The performance of the EGFETs was evaluated using contractile cells (cardiomyocytes).

This paper is organized as follows: first the measuring system is presented. Then the basic working principle of EGFETs is explained, including the fabrication and characterization. Finally, extracellular signals recorded in current as well as in voltage mode are presented and discussed.

\section{Technological Innovation for Cyber-Physical Systems}

Brain-related illnesses affect more than two billion people worldwide. Advances in treatments for brain disorders have to date relied largely upon a pharmaceutical approach, however the development of drugs, which do not have intolerable side effects, is becoming extremely complex and difficult. It is now believed that an electronic engineering-driven approach is needed, to develop solutions based on electrical signals. This is supported by a number of progresses in electronic transducers working as prosthetic and electroceutical devices. These are devices that aim to establish an electrical and chemical bidirectional communication interface with cells and tissues. These devices measure the signals so that researchers can develop a 'dictionary' of patterns associated with health and disease states. Once the signals are decoded, devices can also generate the correct signal patterns to modulate the neural impulses controlling the body, repair lost function and restore health. Field effect transistors based on graphene reported in this contribution are an interesting approach towards the development of these implantable brain-machine interfaces.

\section{Methodology}

\subsection{CVD Growth and Graphene Field Effect Transistor Fabrication and Mouse Embryonic Stem Cells ESC Differentiation}

Graphene has been grown with CVD on copper $(\mathrm{Cu})$ catalyst with $99.99 \%$ purity. The CVD graphene used in this experiment was grown on top of $25 \mu \mathrm{m}$ copper foils (Alfa Aesar, 99.999\% purity). The copper foil was introduced into a thermal CVD system (EasyTube 3000EXT) and annealed at $1020{ }^{\circ} \mathrm{C}$ for 20 minutes in 0.5 Torr hydrogen atmosphere, in order to clean the surface from copper oxides and promote copper grain growth. After that, methane was introduced for 30 minutes under a flow ratio $\mathrm{H}_{2}: \mathrm{CH}_{4}$ of $6: 1$ while keeping the same total pressure, to act as carbon source for the graphene growth. Graphene was then transferred to a final substrate using the copper dissolution method. First, oxygen plasma is used to remove graphene from the bottom side of the copper foil. The other side of the copper is then spin-coated with poly (methyl-2-methylpropenoate) (PMMA, AR-P 679.04) at $1000 \mathrm{rpm}$, followed by a 
bake at $80{ }^{\circ} \mathrm{C}$ for 8 minutes, leading to a nominal PMMA thickness of $630 \mathrm{~nm}$, sufficient to allow manipulation of the membrane without breaking the graphene layer. The copper foil was then dip into a $\mathrm{FeCl}_{3}$ solution until copper was entirely dissolved. The remaining graphene/PMMA membrane, floating in the solution, was then cleaned in an $\mathrm{HCl}$ solution to eliminate iron precipitates and in DI water. At this step grapheme could be transferred onto a final substrate. This substrate was previously prepared by sputter depositing $\mathrm{Cr} 3 \mathrm{~nm} / \mathrm{Au} 30 \mathrm{~nm}$ onto an oxidized silicon substrate, followed by optical lithography and ion milling. Finally, a $320 \mathrm{~nm}$ layer of $\mathrm{Al}_{2} \mathrm{O}_{3}$ was deposited on the substrate by lift-off to provide electrical insulation between the current lines and the electrolyte and a chemical protection. After the graphene/PMMA membrane was transferred onto these substrate from the solution, the substrates were dried for 12 hours at $120^{\circ} \mathrm{C}$ in an oven to enhance graphene adhesion. The PMMA layer then was dissolved in acetone. In order to provide spatial resolution to the cell experiments, each array sample contains several transistors. The photograph in Fig.1a shows one half of a $2 \times 2$ transistor array. The scheme in Fig. 1b shows a cross section view of the device. In our experiments, typical transistors have a channel length $(L)$ of $25 \mu \mathrm{m}$ and a width $(W)$ of $75 \mu \mathrm{m}$.

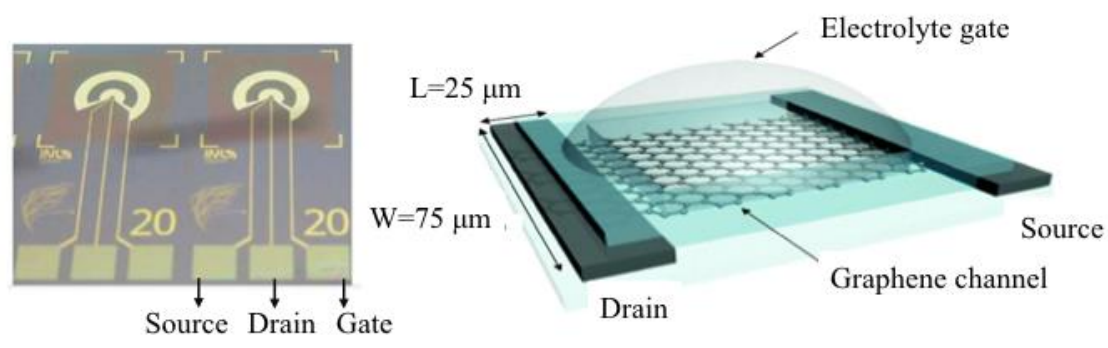

(a)

(b)

Fig. 1. (a) Photograph of two EGFETs on a silicon wafer, (b) schematic diagram showing the drian and source dimensions.

Mouse embryonic stem cells (ESC) differentiated into cardiomyocytes were used. ESC differentiation was performed using the hanging-drop method in medium containing $20 \%$ of fetal bovine serum without Leukemia inhibitory factor (LIF) supplementation (differentiation medium). Briefly, 1000 cells were cultured in $20 \mu 1$ hanging drops of differentiation medium for 48 hours to initiate embryonic bodies (EB) formation. Next, EB were grown in differentiation medium in suspension, for 3 days, 
in a bacterial petri dish before being transferred to the devices $(0.1 \%)$ to allow cell attachment and further differentiation. Cells were kept alive for two days by changing half of the medium every 24 hours. The sensing devices with cells were maintained at $37^{\circ} \mathrm{C}$ in an incubator (HERACell@150) with a humidified atmosphere with $5 \%$ of $\mathrm{CO}_{2}$. A photograph showing the cells on top of a recording device is shown in Fig. 2b.

All electrical measurements were performed with a Stanford low-noise current amplifier (SRS 570) or alternatively in voltage mode using a voltage amplifier (SRS 560) connected to a dynamic signal analyser (Agilent 35670A).

\subsection{Interfacing of Cells with Graphene Based EGFETs}

Figure 2a shows a schematic diagram of the EGFET and the electrical connections. A embryonic body of cardiomyocyte cells was placed on top of the gate terminal (Fig. $2 b)$. Cell activity was measured without biasing the EGFET. No voltages were applied between the drain and source terminals. This measuring method avoids the risk of electrochemical reactions resulting from the use of DC voltages in a liquid environment. The extracellular signals can be detected as a current or as a voltage between the drain and source terminals.

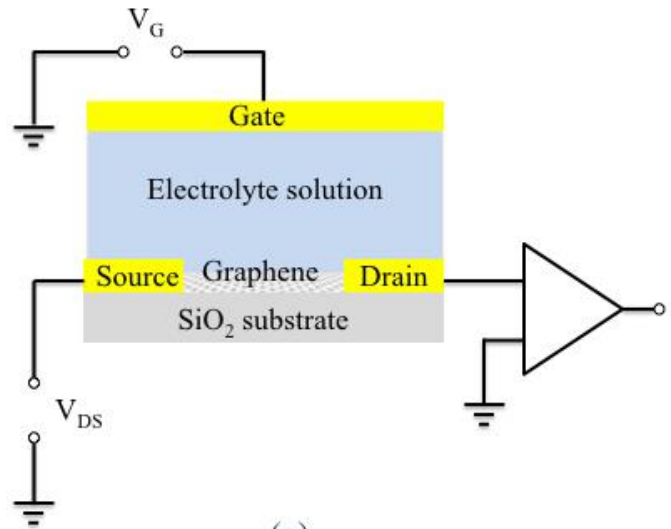

(a)

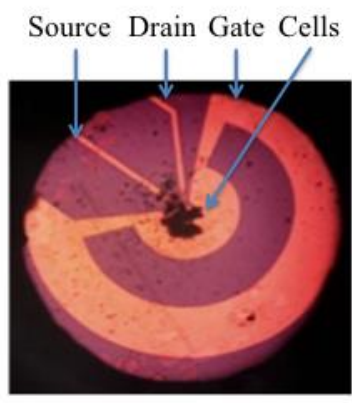

(b)

Fig.2. (a) Schematic diagram showing the electrical connections to the EGFET. Low noise noise amplifiers were used to record the signals in current as well as in voltage, (b) Photograph showing a cluster of cardiomyocytes on top of the gate terminal.

\section{Results and Discussion}

A detail view of the signals in current and in voltage is show in Fig. 3. The spikes in current reach amplitude of 0.4 pico-ampers (peak-to-peak) and voltage signals reach 
values of $28 \mu \mathrm{V}$ (peak-to-peak) in a noise level of $7 \mu \mathrm{V}$. In voltage amplification the signal-to-noise ratio (SNR) reaches 4, a value approximately near the value of 5.6 obtained using current amplification.

Figure 4 shows the thermal noise measured in current of the graphene based EGFET without cells. The noise is represented as a power spectral density (PSD) and it shows a flat noise behavior up to $10 \mathrm{~Hz}$. This noise basically determines the device sensitivity. The origin of noise is not clear yet. Possible sources of noise include charge trapping at the interface to the substrate as well as the so-called charge noise [24,25].

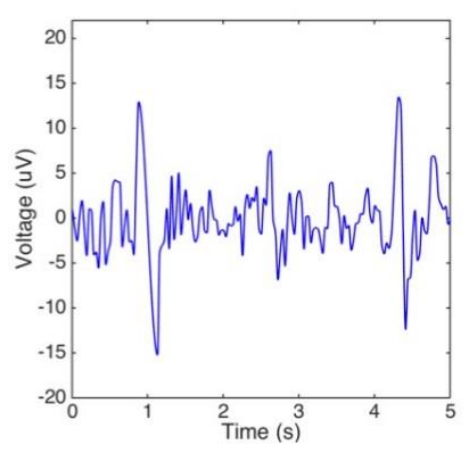

(a)

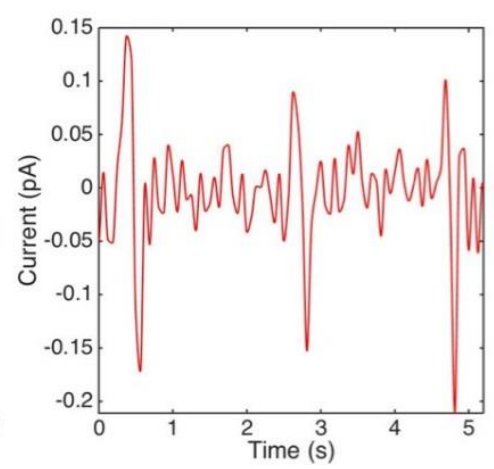

(b)

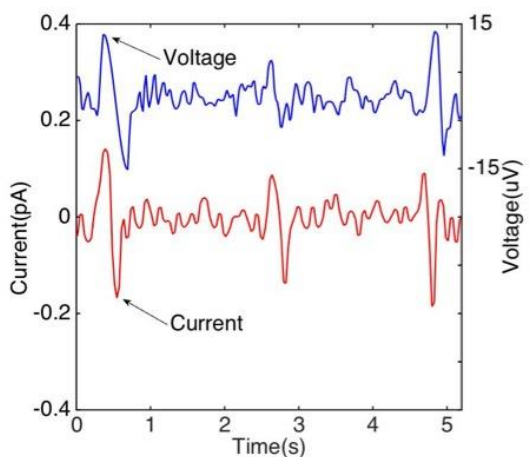

(c)

Fig. 3. Extracellular signals measured as a function of time in (a) voltage mode, (b) current mode, and (c) comparison between voltage and current signals. The voltage signal was shifted along the voltage axis. 


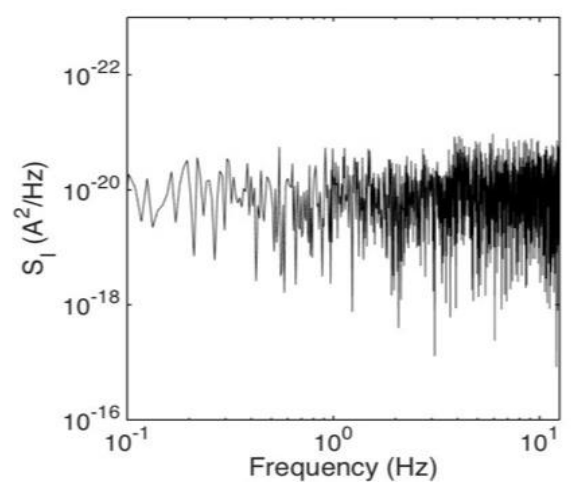

Fig. 4. The noise spectral density (PSD) of the graphene based EGFET without cells.

\section{Conclusion}

This paper reports on the use of a graphene based EGFET to record signals from contractile cells. During electrical measurements the transistor was not biased. In this operation mode the device is working as a simple microelectrode system. This strategy minimizes the electrical noise and the risk of electrochemical reactions. It allows us to explore the detection limits of this type of device. The noise level in current is below 1 pico-amp and in current is below 10 microvolts. The signal-to noise ratio is approximately 5 . This compares very well with the performance of current available microelectrode array technology to measure extracellular signals.

The measured signals are very weak. For contractile cells we would expect a much stronger signals. Among the possible reasons for these faint signals is a bad electrical coupling between the cells and the device. Clusters of cardiomyocytes do not adhere to the device surface. It is well possible that they float above the sensing surface.

\section{References}

1. Fromherz, P,. Offenhausser, A,. Vetter. J. Weis, T.: A neuron-silicon junction: a Retzius cell of the leech on an insulated-gate field-effect transistor. Science 252, 1290 (1991).

2. Timko, B.P., Cohen-Karni, T., Qing, Q., Tian, B., Lieber C.M.: Design and implementation of functional nanoelectronic interfaces with biomolecules, cells, and tissue using nanowire device arrays. Nanotechnology, IEEE Transactions on 9 (3), 269-280 (2010).

3. Kotov, N.A., Winter, J.O., Clements, I.P., Jan, E., Timko, B.P., Campidelli, S., Pathak, S., Mazzatenta, A., Lieber, C.M., Prato, M., Bellamkonda, R.V., Silva, GA., Kam, N.W.S., Patolsky, F., Ballerini, L.: Nanomaterials for neural interfaces. Adv Mater.: 21 (40): 39704004 (2009).

4. Patolsky, F., Timko, B.P., Zheng, G.F., Lieber, CM.: Nanowire-based nanoelectronic devices in the life sciences. MRS Bull; 32:142-149 (2007). 
5. Patolsky, F., Timko, B.P., Yu, G., Fang, Y., Greytak, A.B., Zheng, G., Lieber, C.M.: Detection, stimulation, and inhibition of neuronal signals with high-density nanowire transistor arrays. Science, 313:1100-1104 (2006).

6. Timko, B.P., Cohen-Karni, T., Yu, G., Qing, Q., Tian, B., Lieber, C.M.: Electrical recording from hearts with flexible nanowire device arrays. Nano Lett. 9, 914-918 (2009).

7. Pui, T-S., Agarwal, A., Ye, F., Balasubramanian, N., Chen, P. Small., Eschermann, J.F., Stockmann, R., Hueske, M., Vu, XT., Ingebrandt, S., Offenhäusser, A.: CMOS-Compatible nanowire sensor arrays for detection of cellular bioelectricity. Appl. Phys. Lett. 95:0837031-083703-3 (2009).

8. Cohen-Karni, T., Timko, B.P., Weiss L.E, Lieber C.M.: Flexible electrical recordings from cells using nanowires transistor arrays. Proc. Natl. Acad. Sci USA; 106:7309-7313(2009).

9. Qing, Q., Pal, S.K., Tian, B., Duan, X., Timko, B.P., Cohen-Karni, T., Murthy, V.N., Lieber, CM.: Nanowire transistor arrays for mapping neural circuits in acute brain slices. Proc. Natl. Acad. Sci. USA.0914737107 (2010).

10. Geim, A.K,. Novoselov, K.: The Rise of Graphene. Nature Materials, 6,183-191 (2007).

11. J. Anteroinen, W.Kim., Stadius,K., Riikonen, J., Lipsanen, H., Ryynanen, J.: World Academy of Science, Engineering and Technology, 6 08-25 (2012).

12. Stieglitz, T., Beutel, H., Schuettler, M., Meyer, J.U., Biomed.: Microdevices, 2, 283 (2000).

13. Hess, L. H., Jansen, M., Maybeck, V., Hauf, M. V., Seifert, M., Stutzmann, M., Sharp, I. D., Offenhñusser, A., Garrido, J. A.: Graphene Transistor Arrays for Recording Action Potentials from Electrogenic Cells .Adv. Mater. 23, 5045-5049 (2011).

14. Cohen-karni, T., Li, Q., Fang, Y., Lieber, C.M.: Graphene and nanowire transistors for cellular interfaces and electrical recording. Nano Lett, 10, 1098 (2010).

15. Mohanty, N., Berry, V.: Graphene-Based Single-Bacterium Resolution Biodevice and DNA Transistor: Interfacing Graphene Derivatives with Nanoscale and Microscale Biocomponents. Nano Lett, 8, 4469-4476 (2008).

16. Nguyen, P., Berry, V.: Graphene interfaced with biological cells: opportunities and challenges. The Journal of Physical Chemistry Letters, 3, 1024-1029 (2012).

17. Kim, H., Mattevi, C., Calvo, M. R., Oberg, J.C., Artiglia, L., Agnoli, S., Hirjibehedin, C.F., Chhowalla, M., Saiz, E.: Activation Energy Paths for Graphene Nucleation and Growth on Cu. ACS Nano, 6, 3614-3623 (2012).

18. Mattevi, C., Kim, H., Chhowalla, M.: A review of chemical vapour deposition of graphene on copper. J. Mat. Chem., 21, 3324 (2011).

19. Gomez De Arco, L., Zhang, Y., Schlenker, C.W., Ryu, K., Thompson, M.E., Zhou, C.: Continuous, highly flexible, and transparent graphene films by chemical vapor deposition for organic photovoltaics. ACS Nano, 4, $2865-2873$ (2010).

20. Kim, K.S., Zhao, Y., Jang, H., Lee, S.Y., Kim, J. M., Kim, K.S., Ahn, J.-H., Kim, P., Choi, J.-Y., Hong, B. H.: Large-scale pattern growth of graphene films for stretchable transparent electrodes, Nature, 457, 706-10 (2009).

21. Kim, K., Lee, Z., Regan, W., Kisielowski, C., Crommie, M. F., Zettl, A.: Grain boundary mapping in polycrystalline grapheme. ACS Nano. 2142-2146. 51 (2011).

22. Huang, P.Y., Ruiz-Vargas, C.S., van der Zande, A.M., Whitney, W.S., Levendorf, M.P., Kevek, J.W., Garg, S., Alden, J.S., Hustedt, C.J., Zhu, Y., Park, J., McEuen, P. L., Muller, D.A.: Grains and grain boundaries in single-layer graphene atomic patchworkquilts. Nature, 469, 389-92. 52 (2011).

23. Tapasztó, L., Nemes-Incze, P., Dobrik, G., Jae Yoo, K., Hwang, C., Biró, L.P.: Mapping the electronic properties of individual graphene grain boundaries. Appl. Phys. Lett. 100, 053114 (2012).

24. Lucas, H., Hess, M., Seifert., Garrido, J.A.: Graphene Transistors for Bioelectronics. Proceedings of the IEEE 101 (7), 1780-1792 (2011). 
558 S. Asgarifar at el.

25. Heller, S., Chatoor, J., Männik, M.A.G., Zevenbergen, J.B., Oostinga, A.F., Morpurgo, C., Dekker., S.G. Lemay.: Charge Noise in Graphene Transistors Nano Lett, vol. 10, no. 5, pp. 1563-1567 (2010). 